\title{
CONVERGENCE PROPERTIES OF THE $q$-DEFORMED BINOMIAL DISTRIBUTION
}

\author{
MARTIN ZEINER
}

\begin{abstract}
We consider the $q$-deformed binomial distribution introduced by Jing 1994 and Chung et al. 1995 and establish several convergence results involving the Euler and the exponential distribution; some of them are $q$ analogues of classical results.
\end{abstract}

\section{INTRODUCTION}

The $q$-deformed binomial distribution was introduced by Jing [5] in connection with the $q$-deformed boson oscillator and by Chung et al. [2]. Its probabilites are given by

$$
\mathbb{P}\left(X_{Q D}=x\right)=\left[\begin{array}{l}
n \\
x
\end{array}\right]_{q} \tau^{x}(\tau ; q)_{n-x}, \quad 0 \leq x \leq n, 0 \leq \tau \leq 1,
$$

where

$$
\left[\begin{array}{l}
n \\
x
\end{array}\right]_{q}=\frac{(q ; q)_{n}}{(q ; q)_{x}(q ; q)_{n-x}} \quad \text { and } \quad(z ; q)_{n}=\prod_{i=0}^{n-1}\left(1-z q^{i}\right)
$$

are the $q$-binomial coefficient and the $q$-shifted Pochhammer symbol; an introduction to the $q$-calculus and basic hypergeometric series can be found in Gasper and Rahman [3]. A random variable $X$ which is $q$-deformed binomial distributed let us denote by $X \sim Q D(n, \tau, q)$. Elementary and asymptotic properties are given in Jing [5], Jing and Fan [6], Kemp [10, 11] and in the encyclopaedic book Johnson, Kemp and Kotz [7]. Chung et al. [2] and Kupershmidt [12] gave a representation of the $q$-deformed binomial distribution as a sum of dependent and not identically distributed random variables. One important property is the convergence for fixed paramter $\tau$ and $n \rightarrow \infty$ to an Euler distribution with parameter $\lambda=\tau /(1-q)$, i.e.

$$
\mathbb{P}\left(X_{E}=x\right)=\frac{\lambda^{x}}{(q ; q)_{x}}(-\lambda ; q)_{\infty} .
$$

This is a $q$-analogue of the Poisson distribution since $E((1-q) \lambda) \rightarrow P(\lambda)$ for $q \rightarrow 1$. For properties of this distribution we refer to [7], Kemp [8, 9, 11] and Charalambides and Papadatos [1]. The $q$-deformed binomial distribution is a $q$-analogue of the classical binomial distribution, since in the limit $q \rightarrow 1$ the $q$-deformed binomial distribution with paramter $(n, \tau, q)$ reduces to the binomial distribution with parameters $(n, \tau)$.

This paper is devoted to study sequences of random variables $X_{n}$, where the $X_{n}$ are $Q D\left(n, \tau_{n}, q\right)$-distributed and $\tau_{n}$ converges to a limit $c \in[0,1]$. Section 2 deals with the case $c \in[0,1)$. In particular we show that there is a $q$-analogue of the

Date: February 25, 2009.

2000 Mathematics Subject Classification. Primary: 60F05; Secondary: 05A30, 33D99, 62E15.

Key words and phrases. $q$-deformed binomial distribution, $q$-Poisson distribution, Eulerdistribution, exponential distribution, limit theorems.

M. Zeiner was supported by the NAWI-Graz project and the Austrian Science Fund project S9611 of the National Research Network S9600 Analytic Combinatorics and Probabilistic Number Theory. 
convergence of the classical binomial distribution with constant mean to a Poisson distribution. In Section 3 we examine the case $c=1$. Here the limit distribution can be - depending on the growth rate of the parameters $\tau_{n}$-degenerate, truncated exponential-like or exponential.

\section{Parameter Sequences With Limit $<1$}

In the present section we study sequences of random variables $X_{n}$ which are $Q D\left(n, \tau_{n}, q\right)$-distributed, where the parameters $\tau_{n}$ converge to a $c \in[0,1)$. In particular we prove a $q$-analogue of the convergence of the classical binomial distribution with constant mean to a Poisson distribution.

As noted above the sequence converges in the case of constant parameters $\tau_{n}=\tau$ to an Euler distribution with parameter $\tau /(1-q)$. The following theorem shows that the Euler distribution is the limit distribution for every convergent parameter sequence $\tau_{n}$ with limit in $[0,1)$.

Theorem 2.1. Let $X_{n} \sim Q D\left(n, \tau_{n}, q\right)$. Then, for $n \rightarrow \infty$,

$$
X_{n} \rightarrow E(\tau, q)
$$

if $\tau_{n} \rightarrow \tau$ and $0 \leq \tau<1$.

Proof. Observe that [4, Lemma 2.1] still remains true for $\theta>-1$. Then the theorem follows immediately from the facts $e_{q}(x)=1 / E_{q}(-x)$ and

$$
\left[\begin{array}{l}
n \\
x
\end{array}\right]_{q} \rightarrow \frac{1}{(q ; q)_{x}}
$$

for $n \rightarrow \infty$.

We are now interested in special choices of the parameters $\tau_{n}$ such that the limit $X(q)$ of the sequence $X_{n}(q)$ converges to a Poisson distribution for $q \rightarrow 1$. From the previous theorem we conclude immediately

Theorem 2.2. Let $X_{n} \sim Q D\left(n, \tau_{n}(q), q\right)$ with $\tau_{n}(q) \rightarrow \frac{\lambda}{n}$ for $q \rightarrow 1$ and $\tau_{n}(q) \rightarrow$ $\tau(q)$ for $n \rightarrow \infty$ with the additional property $\frac{\tau(q)}{1-q} \rightarrow \lambda$ in the limit $q \rightarrow 1$. Then the following diagram is commutative:

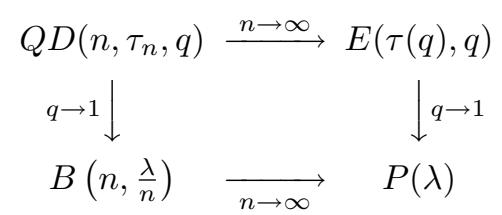

One very natural way to choose the parameters is to set $\tau_{n}=\frac{\lambda}{[n]_{q}}$.

Our next goal is to establish a convergence result, which is analogous to the convergence of the classical binomial distribution with constant mean to a Poisson distribution and reduces in the limit $q \rightarrow 1$ to that theorem. For this purpose we start with an elementary fact.

Lemma 2.3. Let $f_{n}(x), n \in \mathbb{N}$, be a sequence of continuous functions which converge pointwise to a continuous limit $f(x)$. Assume that for each $n$ the function $f_{n}(x)$ has a single root $\hat{x}_{n}$, and $f(x)$ has a single root $\hat{x}$. Then $\hat{x}_{n} \rightarrow \hat{x}$.

Proof. For given $\varepsilon>0$ choose a $\delta(\varepsilon)<\min (f(\hat{x}+\varepsilon),-f(\hat{x}-\varepsilon))$. Then there exists a $N=N(\delta(\varepsilon))$ such that for all $n \geq N$ we have $\left|f_{n}(\hat{x}+\varepsilon)-f(\hat{x}+\varepsilon)\right|<\delta(\varepsilon)$. Therefore $f_{n}(\hat{x}+\varepsilon)>0$. Moreover there exists a $M=M(\delta(\varepsilon))$ such that for all $n \geq M$ we have $\left|f_{n}(\hat{x}-\varepsilon)-f(\hat{x}-\varepsilon)\right|<\delta(\varepsilon)$. Therefore $f_{n}(\hat{x}-\varepsilon)<0$. Hence, by continuity, for all $n \geq \max (N, M)$ we have $\left|\hat{x}-\hat{x}_{n}\right|<2 \varepsilon$. 
The essential key to apply this lemma is the following representation of the means $\mu_{n}(\tau, q)$, which allows us to extract important properties of the means easily.

Lemma 2.4. The means $\mu_{n}(\tau, q)$ have the alternative representation

$$
\mu_{n}(\tau, q)=\sum_{j=1}^{n}(q ; q)_{j-1}\left[\begin{array}{c}
n \\
j
\end{array}\right]_{q} \tau^{j}
$$

Proof. By definition, the means are given by

$$
\mu_{n}(\tau, q)=\sum_{x=1}^{n} x \tau^{x}\left[\begin{array}{l}
n \\
x
\end{array}\right]_{q}(\tau ; q)_{n-x} .
$$

Using the well-known formula (eg. see [3, p. 20])

$$
(z ; q)_{k}=\sum_{i=0}^{k}(-1)^{i}\left[\begin{array}{c}
k \\
i
\end{array}\right]_{q} q^{i(i-1) / 2} z^{i}
$$

we obtain

$$
\mu_{n}(\tau)=\sum_{x=1}^{n} x \tau^{x}\left[\begin{array}{l}
n \\
x
\end{array}\right]_{q} \sum_{i=0}^{n-x}(-1)^{i}\left[\begin{array}{c}
n-x \\
i
\end{array}\right]_{q} q^{i(i-1) / 2} \tau^{i} .
$$

Extracting coefficients in this formula we get

$$
\begin{aligned}
{\left[\tau^{j}\right] \mu_{n}(\tau) } & =\sum_{x=1}^{j} x\left[\begin{array}{l}
n \\
x
\end{array}\right]_{q}(-1)^{j-x}\left[\begin{array}{l}
n-x \\
j-x
\end{array}\right]_{q} q^{(j-x)(j-x-1) / 2} \\
& =\frac{[n]_{q} !}{[n-j]_{q} !} \sum_{x=1}^{j} x(-1)^{j-x} q^{(j-x)(j-x-1) / 2} \frac{1}{[x]_{q} ![j-x]_{q} !} .
\end{aligned}
$$

Now we show by induction that

$$
S(j):=\sum_{x=1}^{j} x(-1)^{j-x} q^{(j-x)(j-x-1) / 2} \frac{[j]_{q} !}{[x]_{q} ![j-x]_{q} !}=(q ; q)_{j-1} .
$$

For $j=1$ this is true. Now suppose that this formula is shown for $1 \leq i \leq j-1$. Using the recursion formula for the binomial coefficients and splitting sums we get

$$
\begin{aligned}
& S(j)=j+\sum_{x=1}^{j-1} x(-1)^{j-x} q^{(j-x)(j-x-1) / 2}\left(\left[\begin{array}{c}
j-1 \\
x
\end{array}\right]_{q} q^{x}+\left[\begin{array}{c}
j-1 \\
x-1
\end{array}\right]_{q}\right) \\
& =j+\sum_{x=1}^{j-1} x(-1)^{j-x} q^{(j-x-1)(j-x-2) / 2+j-x-1+x}\left[\begin{array}{c}
j-1 \\
x
\end{array}\right]_{q} \\
& +\sum_{x=2}^{j-1}(x-1) x(-1)^{j-x} q^{(j-x)(j-x-1) / 2}\left[\begin{array}{l}
j-1 \\
x-1
\end{array}\right]_{q}+\sum_{x=1}^{j-1}(-1)^{j-x} q^{(j-x)(j-x-1) / 2}\left[\begin{array}{l}
j-1 \\
x-1
\end{array}\right]_{q} .
\end{aligned}
$$

Notice that the first sum equals $q^{j-1} S(j-1)$. Shifting indices in the other sums we obtain

$$
\begin{aligned}
S(j)=- & q^{j-1}(q ; q)_{j-2}+(j-1)+\sum_{x=1}^{j-2} x(-1)^{j-x-1} q^{(j-x-1)(j-x-2) / 2}\left[\begin{array}{c}
j-1 \\
x
\end{array}\right]_{q} \\
& +1+\sum_{x=0}^{j-2}(-1)^{j-x-1} q^{(j-x-1)(j-x-2) / 2}\left[\begin{array}{c}
j-1 \\
x
\end{array}\right]_{q} .
\end{aligned}
$$


Now combine the second and third term as well as the fourth and the fifth term to

$$
\begin{aligned}
S(j) & =\left(1-q^{j-1}\right)(q ; q)_{j-2}+\sum_{x=0}^{j-1}(-1)^{j-x-1} q^{(j-x-1)(j-x-2) / 2}\left[\begin{array}{c}
j-1 \\
x
\end{array}\right]_{q} \\
& =(q ; q)_{j-1}+(1 ; q)_{j-1}=(q ; q)_{j-1},
\end{aligned}
$$

where we used formula (1).

Remark 2.5. An alternative way to prove this lemma is to use Kemp's [10, p. 300] representation of the probability generating function, to differentiate and to manipulate the sum.

Using the monotonicity of the $q$-binomial coefficients in $n$ we immediately get

Theorem 2.6. The means $\mu_{n}(\tau, q)$ are strictly increasing in $n$ (for $\tau>0$ ) and $\tau$.

Now we turn to the convergence result:

Theorem 2.7. Fix $\mu>0$. Then the following commutative diagram is valid:

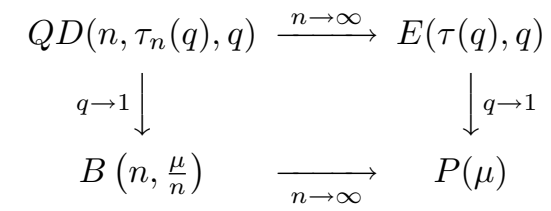

with $\tau_{n}(q)$ determined by $\mu_{n}(\tau, q)=\mu$ and $\tau(q)=\lim _{n \rightarrow \infty} \tau_{n}(q)$.

Proof. First we check, that for given $\mu>0$ and fixed $q$ there exists a unique sequence $\left(\tau_{n}(q)\right)_{n \geq N}$ with $\mu_{n}\left(\tau_{n}(q), q\right)=\mu$. The function $\mu_{n}(\tau, q)$ is continuous and strictly increasing in $n$ and $\tau$ by the previous theorem. Moreover, we have $\lim _{\tau \rightarrow 0} \mu_{n}(\tau, q)=0$. Choosing $\tau_{n}$ in such a way that $\tau_{n}^{n} \rightarrow 1$, then $\mu_{n}\left(\tau_{n}, q\right)$ becomes arbitrary large. Consequently there is a unique solution of $\mu_{n}(\tau, q)=\mu$. By Lemma 2.3 the sequence $\tau_{n}(q)$ converges to a limit $\tau(q)$ where $\tau(q)$ is the unique solution of $\mu_{E}(\tau, q)=\mu$, where $\mu_{E}(\tau, q)$ is the mean of an Euler-distribution mit parameters $\tau$ and $q$. This mean can be written as

$$
\mu_{E}(\tau, q)=\sum_{i=0}^{\infty} \frac{q^{i} \tau}{1-q^{i} \tau},
$$

see [8] or take the limit $n \rightarrow \infty$ in Lemma 2.4 and manipulate the sum.

Again be Lemma 2.3 we get that $\tau(q) \rightarrow \mu / n$. It remains to check that $\tau_{n}(q) /(1-$ $q$ ) converges to $\mu$ in the limit $q \rightarrow 1$. But this is again a consequence of Lemma 2.3 since $\tau(q) /(1-q)$ is the unique solution of $\mu_{E}((1-q) \tau, q)=\mu$ and $\mu_{E}((1-q) \tau, q)$ tends to $\tau$ for $q \rightarrow 1$.

\section{Parameter sequences With Limit 1}

In this section we investigate in sequences $X_{n}$ of random variables, where $X_{n}$ is $Q D\left(n, \tau_{n}, q\right)$-distributed and the parameters $\tau_{n}$ converge to 1 . The behaviour of the sequences $X_{n}$ depends on the growth rate of $\tau_{n}$. Therefor we will distinguish three cases: Firstly we examine the case $\tau_{n}^{n} \rightarrow 1$, where it will turn out that the limit distribution is degenerate. Then we study the case $\tau_{n}^{n} \rightarrow c$ with $0<c<1$. Here the limit distribution will depend only on $c$ and be like a truncated exponential distribution. Finally we turn to the case $\tau_{n}^{f(n)} \rightarrow c$ where $0<c<1$ and $f(n)=$ $o(n)$; this will lead to an exponential distribution.

Consider sequences of random variables $X_{n} \sim Q D\left(n, \tau_{n}, q\right)$ with $\tau_{n} \rightarrow 1$ and additionally $\tau_{n}^{n} \rightarrow 1$ first. Then we have the following theorem: 
Theorem 3.1. Let $X_{n} \sim Q D\left(n, \tau_{n}, q\right)$ with $\tau_{n} \rightarrow 1$ and $\tau_{n}^{n} \rightarrow 1$. Then $n-X_{n}$ converges to the point measure at 0.

Proof. The probability that $Y_{n}=n-X_{n}$ is equal to 0 is given by

$$
\mathbb{P}\left(Y_{n}=0\right)=\tau_{n}^{n}
$$

which converges to 1 by assumption.

Now let us investigate in sequences $X_{n} \sim Q D\left(n, \tau_{n}, q\right)$, where $\tau_{n} \rightarrow 1$ and $\tau_{n}^{n} \rightarrow c$ for a $c \in(0,1)$. Before we can establish the distribution of the limit of such a sequence, we start with several lemmas, which allow us to compute the asymptotic behaviour of certain sums of probabilities of $Q D\left(n, \tau_{n}, q\right)$-distributed random variables and its means and variances.

The first lemma is an analogue to Lemma 2.4 and gives an alternative representation of the variance:

Lemma 3.2. The second moment of $X_{n}(\tau, q)$ can be written as

$$
\sum_{x=1}^{n} x^{2}\left[\begin{array}{l}
n \\
x
\end{array}\right]_{q} \tau^{x}(q ; q)_{n-x}=\sum_{j=1}^{n} a_{j} \tau^{j}
$$

with

$$
a_{j}=\left[\begin{array}{c}
n \\
j
\end{array}\right]_{q}(q ; q)_{j-1}\left(1+2 \sum_{i=1}^{j-1} \frac{1}{1-q^{j-i}}\right)
$$

Proof. Starting as in the proof of Lemma 2.4 we obtain for the coefficients of $\tau^{j}$ in $\mathbb{E}\left(X^{2}\right)$ the formula

$$
\left[\tau^{j}\right] \mathbb{E}\left(X^{2}\right)=\left[\begin{array}{l}
n \\
j
\end{array}\right] \sum_{q=1}^{j} x^{2}(-1)^{j-x} q^{(j-x)(j-x-1) / 2}\left[\begin{array}{l}
j \\
x
\end{array}\right]_{q} .
$$

It remains to show that

$$
T(j):=\sum_{x=1}^{j} x^{2}(-1)^{j-x} q^{(j-x)(j-x-1) / 2}\left[\begin{array}{l}
j \\
x
\end{array}\right]_{q}=(q ; q)_{j-1}\left(1+2 \sum_{i=1}^{j-1} \frac{1}{1-q^{j-i}}\right) .
$$

For this purpose we use induction. For $j=1$ this formula is true. Now suppose that it has been shown for $i \leq j-1$. We use similar ideas as before and write

$$
\begin{aligned}
T(j)= & j^{2}+\sum_{x=1}^{j-1} x^{2}(-1)^{j-x} q^{(j-x)(j-x-1) / 2}\left(\left[\begin{array}{c}
j-1 \\
x
\end{array}\right]_{q} q^{x}+\left[\begin{array}{l}
j-1 \\
x-1
\end{array}\right]_{q}\right) \\
= & j^{2}-q^{j-1} T(j-1)+\sum_{x=2}^{j-1}(x-1)^{2}(-1)^{j-x} q^{(j-x)(j-x-1) / 2}\left[\begin{array}{l}
j-1 \\
x-1
\end{array}\right]_{q} \\
& +\sum_{x=1}^{j-1}(2 x-1)(-1)^{j-x} q^{(j-x)(j-x-1) / 2}\left[\begin{array}{l}
j-1 \\
x-1
\end{array}\right]_{q} .
\end{aligned}
$$

Shifting index in the first sum and adding $(j-1)^{2}$ we obtain

$$
\begin{aligned}
T(j)= & \left(1-q^{j-1}\right) T(j-1)+(2 j-1)+\sum_{x=1}^{j-1}(2 x-1)(-1)^{j-x} q^{(j-x)(j-x-1) / 2} \\
= & \left(1-q^{j-1}\right) T(j-1)+2(j-1)+2 \sum_{x=0}^{j-2} x(-1)^{j-x-1} q^{(j-x-1)(j-x-2) / 2}\left[\begin{array}{c}
j-1 \\
x
\end{array}\right]_{q} \\
& +1+\sum_{x=0}^{j-2}(-1)^{j-x-1} q^{(j-x-1)(j-x-2) / 2}\left[\begin{array}{c}
j-1 \\
x
\end{array}\right]_{q} .
\end{aligned}
$$


Now we use the proof of Lemma 2.4 again and induction hypothesis to get

$$
\begin{aligned}
T(j) & =\left(1-q^{j-1}\right) T(j-1)+2 S(j-1) \\
& =\left(1-q^{j-1}\right)(q ; q)_{j-2}\left(1+2 \sum_{i=1}^{j-2} \frac{1}{1-q^{j-i-1}}\right)+2(q ; q)_{j-2} \\
& =(q ; q)_{j-1}\left(1+2 \sum_{i=1}^{j-2} \frac{1}{1-q^{j-i-1}}+\frac{2}{1-q^{j-1}}\right) \\
& =(q ; q)_{j-1}\left(1+2 \sum_{i=1}^{j-1} \frac{1}{1-q^{j-i}}\right)
\end{aligned}
$$

which completes the proof.

The next three lemmas are devoted to the asymptotic behaviour of sums of powers of $\theta_{n}$, where $0<\theta_{n}<1$ and $\theta_{n} \rightarrow 1$.

Lemma 3.3. If $f(n) \rightarrow \infty$ for $n \rightarrow \infty$ and $\theta_{n} \leq 1$ such that $\theta_{n}^{f(n)} \rightarrow c$ with $0<c<1$, then

$$
\sum_{i=0}^{\infty} \theta_{n}^{i} \sim \frac{-f(n)}{\log c}, \quad n \rightarrow \infty .
$$

Proof. We use Euler-MacLaurin's formula and obtain

$$
\begin{aligned}
\sum_{i=0}^{\infty} \theta_{n}^{i} & =\int_{0}^{\infty} \theta_{n}^{x} \mathrm{~d} x+\frac{1}{2}+\log \theta_{n} \int_{0}^{\infty}\left(\{x\}-\frac{1}{2}\right) \theta_{n}^{x} \mathrm{~d} x \\
& =\frac{-1}{\log \theta_{n}}+\frac{1}{2}+\log \theta_{n} \int_{0}^{\infty}\left(\{x\}-\frac{1}{2}\right) \theta_{n}^{x} \\
& =\frac{-f(n)}{\log \theta_{n}^{f(n)}}+\frac{1}{2}+\log \theta_{n} \int_{0}^{\infty}\left(\{x\}-\frac{1}{2}\right) \theta_{n}^{x} \sim \frac{-f(n)}{\log c},
\end{aligned}
$$

since the term with the integral is bounded.

Lemma 3.4. For $\theta_{n} \leq 1$ and $\theta_{n} \rightarrow 1, \theta_{n}^{f(n)} \rightarrow c$ for $a c \in(0,1)$ and $g(n) / f(n) \sim \beta$, $g(n) \leq n$ we have

$$
\sum_{i=0}^{\lfloor g(n)\rfloor} \theta_{n}^{i} \sim \frac{c^{\beta}-1}{\log c} f(n)
$$

and

$$
\sum_{i=0}^{\lfloor g(n)\rfloor}\left[\begin{array}{l}
n \\
i
\end{array}\right]_{q} \theta_{n}^{i} \sim e_{q}(q) \frac{c^{\beta}-1}{\log c} f(n)
$$

as $n \rightarrow \infty$.

Proof. For the first sum, we rewrite this sum as

$$
\sum_{i=0}^{\lfloor g(n)\rfloor} \theta_{n}^{i}=\frac{1-\theta_{n}^{\lfloor g(n)\rfloor+1}}{1-\theta_{n}} .
$$

The growth of the denominator is given in Lemma 3.3, and the numerator tends to $1-c^{\beta}$, since $\theta_{n}^{\lfloor g(n)\rfloor}=\theta_{n}^{g(n)-\{g(n)\}} \rightarrow c^{\beta}$ because of $\theta_{n} \rightarrow 1$. 
To get the asymptotic of the second sum we write

$$
\sum_{i=0}^{\lfloor g(n)\rfloor}\left[\begin{array}{c}
n \\
i
\end{array}\right]_{q} \theta_{n}^{i}=\sum_{i=0}^{\lfloor\sqrt{g(n)}\rfloor}\left[\begin{array}{c}
n \\
i
\end{array}\right]_{q} \theta_{n}^{i}+\sum_{\lfloor\sqrt{g(n)}\rfloor+1}^{\lfloor g(n)-\sqrt{g(n)}\rfloor-1}\left[\begin{array}{c}
n \\
i
\end{array}\right]_{q} \theta_{n}^{i}+\sum_{\lfloor g(n)-\sqrt{g(n)}\rfloor}^{\lfloor g(n)\rfloor}\left[\begin{array}{c}
n \\
i
\end{array}\right]_{q} \theta_{n}^{i} .
$$

The first and the third sum on the right-hand side are $\mathcal{O}(\sqrt{g(n)})$ and therefore asymptotically negligible. The second sum ist bounded by

$$
\begin{gathered}
\frac{(q ; q)_{n}}{(q ; q)_{\lfloor\sqrt{g(n)}\rfloor+1}(q ; q)_{n-\lfloor\sqrt{g(n)}\rfloor-1}} \sum_{\lfloor\sqrt{g(n)}\rfloor+1}^{\lfloor g(n)-\sqrt{g(n)}\rfloor-1} \theta_{n}^{i} \leq \sum_{\lfloor\sqrt{g(n)}\rfloor+1}^{\lfloor g(n)-\sqrt{g(n)}\rfloor-1}\left[\begin{array}{c}
n \\
i
\end{array}\right]_{q} \theta_{n}^{i} \\
\leq \frac{(q ; q)_{n}}{\left.(q ; q)_{\lfloor n / 2}^{2}\right\rfloor} \sum_{\lfloor\sqrt{g(n)}\rfloor+1}^{\lfloor g(n)-\sqrt{g(n)}\rfloor-1} \theta_{n}^{i} .
\end{gathered}
$$

By the first part of this lemma the lower and the upper bound has the asserted asymptotic.

Lemma 3.5. If $\theta_{n} \leq 1$ and $\theta_{n} \rightarrow 1$ with $\theta_{n}^{n} \rightarrow c$ for $0<c<1$, then

$$
\sum_{i=0}^{n} i \theta_{n}^{i} \sim \frac{1-c+c \log c}{\log ^{2} c} n^{2}
$$

and

as $n \rightarrow \infty$.

$$
\sum_{i=0}^{n}\left[\begin{array}{c}
n \\
i
\end{array}\right]_{q} i \theta_{n}^{i} \sim e_{q}(q) \frac{1-c+c \log c}{\log ^{2} c} n^{2}
$$

Proof. To estimate this sum we use Lemma 3.3 again and the identity

$$
\sum_{i=0}^{n} i t^{i}=\frac{t\left(1-t^{n}-n t^{n}(1-t)\right)}{(1-t)^{2}}
$$

Hence, setting $t=\theta_{n}$,

$$
\sum_{i=0}^{n} i \theta_{n}^{i} \sim\left(1-c-n \theta_{n}^{n}\left(1-\theta_{n}\right)\right) \frac{n^{2}}{\log ^{2} c} \sim(1-c+c \log c) \frac{n^{2}}{\log ^{2} c} .
$$

Here we used that under the assumption $\tau_{n}^{n} \rightarrow c$ we have $\left(1-\tau_{n}\right) n \rightarrow-\log c$. To see this, write

Then

$$
\tau_{n}=c^{1 / n}+f(n)
$$

$$
\tau_{n}^{n}=c+\sum_{i=1}^{n}\left(\begin{array}{c}
n \\
i
\end{array}\right) f(n)^{i} c^{\frac{n-i}{n}}=: c+s_{n} .
$$

Now suppose for a moment $f(n) \geq 0$. The sum $s_{n}$ must tend to 0 and we have $\left|s_{n}\right|=s_{n} \geq f(n) n c$. Therefore $f(n) n \rightarrow 0$. Similarly, $f(n) n \rightarrow 0$ for negative $f$. Consequently $f(n) n \rightarrow 0$ for arbitrary $f$. Now consider

$$
\left(1-\tau_{n}\right) n=n\left(1-c^{1 / n}\right)+n f(n) \rightarrow-\log c .
$$

The asymptotic for the sum with the $q$-binomial coefficient is obtained as in Lemma 3.4.

Now we are ready to establish the essential key in proving the convergence result: we give the asymptotic behaviour of sums of probabilities and the means and variances of $Q D\left(n, \tau_{n}, q\right)$-distributed random variables. 
Lemma 3.6. Let $X_{n}$ be $Q D\left(n, \tau_{n}, q\right)$-distributed and denote with $\mu_{n}\left(\tau_{n}, q\right)$ and $\sigma_{n}^{2}\left(\tau_{n}, q\right)$ the corresponding mean and variance. If $\tau_{n} \rightarrow 1$ and $\tau_{n}^{n} \rightarrow c$ with $0<$ $c<1$ and $f(n) \sim \beta n, f(n)<n$, then

$$
\begin{aligned}
\sum_{x=0}^{\lfloor f(n)\rfloor} \tau_{n}^{x}\left[\begin{array}{l}
n \\
x
\end{array}\right]_{q}\left(\tau_{n} ; q\right)_{n-x} & \sim 1-c^{\beta} \\
\mu_{n}\left(\tau_{n}, q\right) & \sim \frac{c-1}{\log c} n \\
\sigma_{n}^{2}\left(\tau_{n}, q\right) & \sim \frac{1+2 c \log c-c^{2}}{(\log c)^{2}} n^{2}
\end{aligned}
$$

as $n \rightarrow \infty$.

Proof. We start the first assertion. Since $f(n)<n$ we can write

$$
S_{n}:=\sum_{x=0}^{\lfloor f(n)\rfloor} \tau_{n}^{x}\left[\begin{array}{l}
n \\
x
\end{array}\right]_{q}\left(\tau_{n} ; q\right)_{n-x}=\left(1-\tau_{n}\right) \sum_{x=0}^{\lfloor f(n)\rfloor} \tau_{n}^{x}\left[\begin{array}{l}
n \\
x
\end{array}\right]_{q} \prod_{i=1}^{n-x-1}\left(1-\tau_{n} q^{i}\right) .
$$

The summands are bounded by $e_{q}(q)^{2}$, hence

$$
S_{n} \sim\left(1-\tau_{n}\right) \sum_{x=\lfloor\sqrt{n}\rfloor}^{\lfloor f(n)\rfloor-\lfloor\sqrt{n}\rfloor} \tau_{n}^{x}\left[\begin{array}{l}
n \\
x
\end{array}\right]_{q} \prod_{i=1}^{n-x-1}\left(1-\tau_{n} q^{i}\right)=: \hat{S}_{n}
$$

Estimating the product and using again the boundedness of the summands yields

$$
\begin{aligned}
\hat{S}_{n} & \leq\left(1-\tau_{n}\right)\left(\tau_{n} ; q\right)_{n-\lfloor f(n)\rfloor+\lfloor\sqrt{n}\rfloor-1} \sum_{x=\lfloor\sqrt{n}\rfloor}^{\lfloor f(n)\rfloor-\lfloor\sqrt{n}\rfloor} \tau_{n}^{x}\left[\begin{array}{l}
n \\
x
\end{array}\right]_{q} \\
& \sim\left(1-\tau_{n}\right)\left(\tau_{n} ; q\right)_{n-\lfloor f(n)\rfloor+\lfloor\sqrt{n}\rfloor-1} \sum_{1}^{n} \tau_{n}^{x}\left[\begin{array}{l}
n \\
x
\end{array}\right]_{q}=: \hat{\hat{S}}_{n} .
\end{aligned}
$$

Since [4, Lemma 2.1] remains true for $\theta_{n}>\varepsilon>-1$ and with use of Lemma 3.4 (with $g(n):=f(n)$ and $f(n):=n$ ) we obtain

$$
\hat{\hat{S}}_{n} \sim\left(1-\tau_{n}\right) \frac{1}{e_{q}(q)} e_{q}(q) \frac{c^{\beta}-1}{\log c} n \sim 1-c^{\beta} .
$$

In an analogous way we find a lower bound of $\hat{S}_{n}$ that is asymptotic equivalent to $1-c^{\beta}$.

Now we prove the second proposition of the lemma: Use Lemma 2.4, easy estimates of the $q$-Pochhammer symbol and the asymptotics given in Lemma 3.4 to obtain

$$
\mu_{n}\left(\tau_{n}, q\right) \leq \sum_{j=1}^{\lfloor\sqrt{n}\rfloor} \frac{(q ; q)_{n}}{(q ; q)_{\lceil n / 2\rceil}^{2}}+(q ; q)_{\lfloor\sqrt{n}\rfloor} \sum_{j=\lfloor\sqrt{n}\rfloor}^{n}\left[\begin{array}{c}
n \\
j
\end{array}\right]_{q} \tau_{n}^{j} \sim \frac{1}{e_{q}(q)} e_{q}(q) \frac{c-1}{\log c} n
$$

and

$$
\mu_{n}\left(\tau_{n}, q\right) \geq(q ; q)_{n} \sum_{j=1}^{n}\left[\begin{array}{l}
n \\
j
\end{array}\right]_{q} \tau_{n}^{j} \sim \frac{c-1}{\log c} n .
$$

Similarly we proceed for the second moments of $X_{n}\left(\tau_{n}, q\right)$ and estimate with use of Lemma 3.5

$\mathbb{E}\left(X_{n}^{2}\right) \geq \sum_{j=1}^{n}(q ; q)_{j-1}(1+2(j-1))\left[\begin{array}{l}n \\ j\end{array}\right]_{q} \tau_{n}^{j} \geq 2(q ; q)_{n} \sum_{j=1}^{n}(j-1)\left[\begin{array}{c}n \\ j\end{array}\right]_{q} \tau_{n}^{j} \sim 2 \frac{1-c+c \log c}{(\log c)^{2}} n^{2}$. 
To bound the second moment from above we do the following:

$$
\begin{aligned}
& \mathbb{E}\left(X_{n}^{2}\right) \leq \sum_{j=1}^{\lfloor\sqrt{n}\rfloor} \frac{(q ; q)_{n}}{(q ; q)_{\lceil n / 2\rceil}^{2}}\left(1+\frac{2 n}{1-q}\right)+\sum_{j=\lfloor\sqrt{n}\rfloor}^{n}(q ; q)_{j-1}\left(1+2 \sum_{i=1}^{j-1} \frac{1}{1-q^{j-i}}\right)\left[\begin{array}{c}
n \\
j
\end{array}\right]_{q} \tau_{n}^{j} \\
& =o\left(n^{2}\right)+\sum_{j=\lfloor\sqrt{n}\rfloor}^{n}(q ; q)_{j-1}\left(1+2 \sum_{i=\lfloor\sqrt{j}\rfloor}^{j-1} \frac{1}{1-q^{j-i}}\right)\left[\begin{array}{c}
n \\
j
\end{array}\right]_{q} \tau_{n}^{j} \\
& +\sum_{j=\lfloor\sqrt{n}\rfloor}^{n}(q ; q)_{j-1}\left(1+2 \sum_{i=1}^{\lfloor\sqrt{j}\rfloor} \frac{1}{1-q^{j-i}}\right)\left[\begin{array}{c}
n \\
j
\end{array}\right]_{q} \tau_{n}^{j} \\
& \leq o\left(n^{2}\right)+2(q ; q)_{\lfloor\sqrt{n}\rfloor} \sum_{j=\lfloor\sqrt{n}\rfloor}^{n} j \frac{1}{1-q^{j-\lfloor\sqrt{j}\rfloor-1}}\left[\begin{array}{c}
n \\
j
\end{array}\right]_{q} \tau_{n}^{j} \\
& \leq o\left(n^{2}\right)+2(q ; q)_{\lfloor\sqrt{n}\rfloor} \frac{1}{1-q^{n-\sqrt{n}-1}} \sum_{j=1}^{n} j\left[\begin{array}{l}
n \\
j
\end{array}\right]_{q} \tau_{n}^{j} \\
& \sim 2 \frac{1-c+c \log c}{(\log c)^{2}} n^{2} .
\end{aligned}
$$

Thus

$$
\mathbb{E}\left(X_{n}^{2}\left(\tau_{n}, q\right)\right) \sim 2 \frac{1-c+c \log c}{(\log c)^{2}} n^{2}
$$

Hence

$$
\begin{aligned}
\sigma_{n}^{2}\left(\tau_{n}, q\right) & =\mathbb{E}\left(X_{n}^{2}\left(\tau_{n}, q\right)\right)-\mu_{n}(\tau, q)^{2} \sim\left(2 \frac{1-c+c \log c}{(\log c)^{2}}-\left(\frac{c-1}{\log c}\right)^{2}\right) n^{2} \\
& \sim \frac{1+2 c \log c-c^{2}}{(\log c)^{2}} n^{2}
\end{aligned}
$$

After this anaysis of the means and variances it is now easy to obtain the limiting distribution of the sequence $X_{n}$.

Theorem 3.7. Let $Y_{n} \sim Q D\left(n, q, \tau_{n}\right)$ with $\tau_{n} \rightarrow 1$ and $\tau_{n}^{n} \rightarrow c$ with $0<c<1$. Then the sequence of the normalised random variables $X_{n}=\left(Y_{n}-\mu_{n}\right) / \sigma_{n}$ converges to a limit $X$ with

$$
\mathbb{P}(X \leq x)=1-e^{c-1} e^{-\sqrt{1+2 c \log c-c^{2}} x}
$$

for

and

$$
x \in\left[-\frac{1-c}{\sqrt{1+2 c \log c-c^{2}}}, \frac{c-\log c-1}{\sqrt{1+2 c \log c-c^{2}}}\right)
$$

$$
\mathbb{P}(x)=1 \quad \text { for } \quad x=\frac{c-\log c-1}{\sqrt{1+2 c \log c-c^{2}}} .
$$

Proof. The support of $X$ is given by

$$
\left[\lim _{n \rightarrow \infty}-\frac{\mu_{n}\left(\tau_{n}, q\right)}{\sigma_{n}\left(\tau_{n}, q\right)}, \lim _{n \rightarrow \infty} \frac{n-\mu_{n}\left(\tau_{n}, q\right)}{\sigma_{n}\left(\tau_{n}, q\right)}\right] .
$$

Using Lemma 3.6 the stated support follows immediately.

Computing the distribution function of $X$ yields with use of Lemma 3.6

$$
\mathbb{P}\left(X_{n} \leq x\right)=\sum_{0 \leq y \leq \sigma_{n} x+\mu_{n}} \tau_{n}^{y}\left[\begin{array}{l}
n \\
y
\end{array}\right]_{q}\left(\tau_{n} ; q\right)_{n-y} \sim 1-c^{\alpha}
$$


with

for

$$
\alpha=\frac{\sqrt{1+2 c \log c-c^{2}}}{-\log c} x+\frac{c-1}{\log c}
$$

$$
x<\frac{c-\log c-1}{\sqrt{1+2 c \log c-c^{2}}} .
$$

Simplifying $c^{\alpha}$ yields the theorem.

Now we turn to the third case, which treats with sequences of random variables $X_{n} \sim Q D\left(n, \tau_{n}, q\right)$ where $\tau_{n} \rightarrow 1$ and $\tau_{n}^{f(n)} \rightarrow c$ for a $c \in(0,1)$ and $f(n)=o(n)$. This case is very similar to the previous one, and so we start with an analogue of Lemma 3.5

Lemma 3.8. Let $f(n) \rightarrow \infty, f(n)=o(n), \theta_{n}^{f(n)} \rightarrow c$ with $0<c<1$. Then

$$
\sum_{i=0}^{n} i \theta_{n}^{i} \sim \frac{f(n)^{2}}{\log ^{2} c} \quad \text { and } \quad \sum_{i=0}^{n} i\left[\begin{array}{l}
n \\
i
\end{array}\right]_{q} \theta_{n}^{i} \sim e_{q}(q) \frac{f(n)^{2}}{\log ^{2} c}
$$

as $n \rightarrow \infty$.

Proof. Follow the proof of Lemma 3.5 and observe that $n \theta_{n}^{n}\left(1-\theta_{n}\right)$ tends to zero.

Following the proof of Lemma 3.6 and using Lemma 3.8 instead of Lemma 3.5 we obtain

Lemma 3.9. If $\tau_{n} \rightarrow 1$ and $\tau_{n}^{f(n)} \rightarrow c$ with $0<c<1$ and $f(n)=o(n), g(n) \sim$ $\beta f(n)$, then

$$
\begin{aligned}
\sum_{x=0}^{\lfloor g(n)\rfloor} \tau_{n}^{x}\left[\begin{array}{l}
n \\
x
\end{array}\right]_{q}\left(\tau_{n} ; q\right)_{n-x} & \sim 1-c^{\beta} \\
\mu_{n}\left(\tau_{n}, q\right) & \sim \frac{-f(n)}{\log c} \\
\sigma_{n}^{2}\left(\tau_{n}, q\right) & \sim \frac{f(n)^{2}}{(\log c)^{2}}
\end{aligned}
$$

as $n \rightarrow \infty$.

As an immediate consequence we get the distribution of the limit of $X_{n}$, which is an exponential distribution and is again independent of $q$.

Theorem 3.10. Let $Y_{n} \sim Q D\left(n, q, \tau_{n}\right)$ with $\tau_{n} \rightarrow 1$ and $\tau_{n}^{f(n)} \rightarrow c$ with $0<c<1$ and $f(n)=o(n)$. Then the sequence of the normalised random variables $X_{n}=$ $\left(Y_{n}-\mu_{n}\right) / \sigma_{n}$ converges to a normalised exponential distribution with parameter 1 , i.e.

$$
\mathbb{P}(X \leq x)=1-e^{-x-1}, \quad x \geq-1 .
$$

Proof. Lemma 3.9 yields immediately that the support of the limit distribution is $[-1, \infty)$. Computing the distribution function gives

$$
\mathbb{P}(X \leq x)=\sum_{0 \leq y \leq \sigma_{n} x+\mu_{n}} \tau_{n}^{y}\left[\begin{array}{l}
n \\
y
\end{array}\right]_{q}\left(\tau_{n} ; q\right)_{n-y} \sim 1-c^{\frac{x+1}{-\log c}}=1-e^{-x-1} .
$$

Comparing this result with Theorem 3.7 we see that this corresponds to taking the limit $c \rightarrow 0$. 


\section{REFERENCES}

[1] C. A. Charalambides And N. Papadatos, The q-factorial moments of discrete qdistributions and a characterization of the Euler distribution, in Advances on models, characterizations and applications, vol. 180 of Stat. Textb. Monogr., Chapman \& Hall/CRC, Boca Raton, FL, 2005, pp. 57-71.

[2] W.-S. Chung, K.-S. Chung, H.-J. Kang, And N.-Y. ChoI, q-deformed probability and binomial distribution, Internat. J. Theoret. Phys., 34 (1995), pp. 2165-2170.

[3] G. Gasper and M. Rahman, Basic hypergeometric series, vol. 35 of Encyclopedia of Mathematics and its Applications, Cambridge University Press, Cambridge, 1990. With a foreword by Richard Askey.

[4] S. Gerhold And M. Zeiner, Convergence properties of Kemp's q-binomial distribution. preprint.

[5] S. C. JING, The q-deformed binomial distribution and its asymptotic behaviour, J. Phys. A, 27 (1994), pp. 493-499.

[6] S. C. Jing And H. Y. FAn, q-deformed binomial state, Phys. Rev. A (3), 49 (1994), pp. 22772279.

[7] N. L. Johnson, A. W. Kemp, And S. Kotz, Univariate discrete distributions, Wiley Series in Probability and Statistics, Wiley-Interscience [John Wiley \& Sons], Hoboken, NJ, third ed., 2005.

[8] A. W. Kemp, Heine-Euler extensions of the Poisson distribution, Comm. Statist. Theory Methods, 21 (1992), pp. 571-588.

[9] - Steady-state Markov chain models for the Heine and Euler distributions, J. Appl. Probab., 29 (1992), pp. 869-876.

[10] - Certain q-analogues of the binomial distribution, Sankhyā Ser. A, 64 (2002), pp. 293305. Selected articles from San Antonio Conference in honour of C. R. Rao (San Antonio, TX, 2000).

[11] - Characterizations involving $U \mid(U+V=m)$ for certain discrete distributions, J. Statist. Plann. Inference, 109 (2003), pp. 31-41.

[12] B. A. Kupershmidt, q-probability. I. Basic discrete distributions, J. Nonlinear Math. Phys., 7 (2000), pp. 73-93.

(Martin Zeiner) Graz University of Technology, Department for Analysis and Computational Number Theory, Steyrergasse 30/II, 8010 Graz, Austria

E-mail address: zeiner at finanz.math.tu-graz.ac.at 